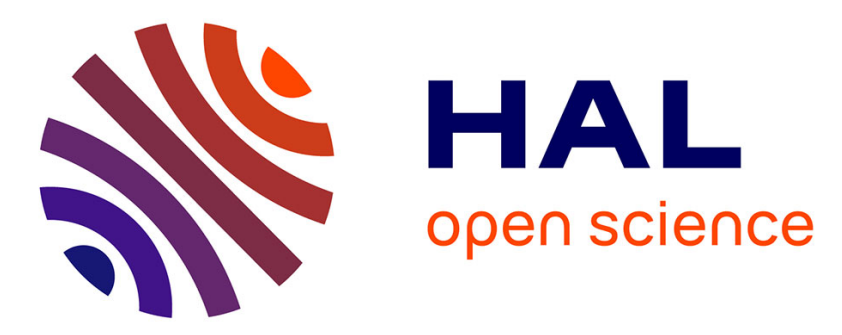

\title{
The bi-potential method applied for the modeling of dynamic problems with friction
}

Zhi-Qiang Feng, Pierre Joli, Jean-Michel Cros, Benoit Magnain

\section{To cite this version:}

Zhi-Qiang Feng, Pierre Joli, Jean-Michel Cros, Benoit Magnain. The bi-potential method applied for the modeling of dynamic problems with friction. Computational Mechanics, 2005, 36 (5), pp.375-383. 10.1007/s00466-005-0663-8 . hal-00342921

\section{HAL Id: hal-00342921 \\ https://hal.science/hal-00342921}

Submitted on 25 Jun 2018

HAL is a multi-disciplinary open access archive for the deposit and dissemination of scientific research documents, whether they are published or not. The documents may come from teaching and research institutions in France or abroad, or from public or private research centers.
L'archive ouverte pluridisciplinaire $\mathbf{H A L}$, est destinée au dépôt et à la diffusion de documents scientifiques de niveau recherche, publiés ou non, émanant des établissements d'enseignement et de recherche français ou étrangers, des laboratoires publics ou privés. 


\section{The bi-potential method applied to the modeling of dynamic problems with friction}

\begin{abstract}
The bi-potential method has been successfully applied to the modeling of frictional contact problems in static cases. This paper presents an extension of this method for dynamic analysis of impact problems with deformable bodies. A first order algorithm is applied to the numerical integration of the time-discretized equation of motion. Using the Object-Oriented Programming (OOP) techniques in $\mathrm{C}++$ and OpenGL graphical support, a finite element code including pre/postprocessor FER/Impact is developed. The numerical results show that, at the present stage of development, this approach is robust and efficient in terms of numerical stability and precision compared with the penalty method.
\end{abstract}

Keywords Impact $\cdot$ Bi-potential method $\cdot$ Finite element method $\cdot$ Time-integration

\section{Introduction}

Problems involving contact and friction are the most difficult ones in mechanics and at the same time of crucial and practical significance in many engineering branches. The main mathematical difficulty lies in the severe contact non-linearities because the natural first order constitutive laws of contact and friction phenomena are expressed by non-smooth multivalued forcedisplacement or force-velocity relations. In the past decade, substantial progress has been made in the analysis of contact problems using finite element procedures. A large number of algorithms for the numerical solution to the related finite element equations and inequalities have been presented in the literature. Re-

Z.-Q. Feng Æ P. Joli Æ J.-M. Cros Æ B. Magnain Laboratoire de Mécanique et d'Energétique d'Evry (LMEE), Université d'Evry Val d'Essonne, 40 rue du Pelvoux, 91020 Evry cedex, France

E-mail: feng@iup.univ-evry.fr views may be consulted for an extensive list of references [1-3]. Also see the monographs by Kikuchi and Oden [4], Zhong [5] and Wriggers [6]. The popular penalty approximation [7] and 'mixed' or 'trial-and-error' methods appear, at first glance, suitable for many applications. But in this kind of method, the contact boundary conditions and friction laws are not satisfied accurately and it is tricky for the users to choose appropriate penalty factors. They may fail for stiff problems because of unpleasant numerical oscillations among contact statuses [8]. The augmented Lagrangian method first appeared to deal with constrained minimization problems. Since friction problems are not minimum problems, the formulation needs to be extended. Alart and Curnier [9], Simo and Laursen [10] and De Saxcé and Feng [11] have obtained some extensions in mutually independent works. Alart and Curnier applied Newton's method to the saddle-point equations of the augmented Lagrangian. Simo and Laursen developed an augmented Lagrangian formulation and a Uzawa type scheme for frictional contact problems. De Saxcé and Feng proposed a theory called ISM (Implicit Standard Materials) and a bi-potential method, in which another augmented Lagrangian formulation was developed, which is different from that of the first two works. In particular, in the bi-potential method, the frictional contact problem is treated in a reduced system by means of a reliable and efficient predictor-corrector solution algorithm. For the unilateral contact problems with friction, the classic approach is based on two minimum principles or two variational inequalities: the first for unilateral contact and the second for friction. The bipotential method leads to a single displacement variational principle and a unique inequality. In consequence, the unilateral contact and the friction are coupled via a contact bi-potential. The application of the augmented Lagrangian method to the contact laws leads to an equation of projection onto Coulomb's cone, strictly equivalent to the original inequality [12]. For additional comments, also see the interesting discussion by Klarbring et al. [13, 14]. 
For dynamic implicit analysis in structural mechanics, the most commonly used integration algorithm is the second order algorithm such as Newmark, Wilson, etc. Armero and Petocz [15] and Laursen and Chawla [16] have considered frictionless dynamic impact under the auspices of a conservative system, and have proposed the means to address the dynamic contact conditions so that they preserve the global conservation properties. Armero and Petocz [15] presume a penalty enforcement of the contact constraint, algorithmically preserving the energy dissipation associated with a new contact event and restoring it to the system upon release. Laursen and Chawla [16] also choose to concede an interpenetration of the contact surfaces in order to establish a Lagrange multiplier solution for each contact time step with energy conservation. In both cases, the contact constraints have been modified in pursuit of the conservation properties, resulting in an incomplete enforcement of what might be considered normal geometric constraints (i.e. impenetrability). Recently, Laursen and Love [17] have proposed an improved implicit integration scheme with a velocity update algorithm to avoid the interpenetration of the contact surfaces. It is well known that in impact problems, the velocity and acceleration are not continuous because of sudden changes in contact conditions (impact, release of contact). So the second order algorithms with regularity constraints may lead to serious errors. To avoid this shortcoming, some first order algorithms have been proposed by Zienkiewicz et al. [18], Moreau [19], Jean and Wronski [20, 21].

The aim of the present paper is to extend the bipotential method for contact modeling in dynamic cases in the field of Non-Smooth Dynamics using the first order algorithm for integration of the equation of motion. The developed algorithm is implemented into the code FER/Impact, using $\mathrm{C}++$ with object oriented programming techniques and OpenGL graphical support. Two numerical examples are performed in this study to show the validity of the model developed. The first example concerns the oblique impact of a 2D elastic plate onto a rigid surface with rebounding. The performance of FER/Impact is reported as compared to the general purpose finite element code ANSYS in which the penalty method is used for contact modeling [22, 23]. The second example simulates the impact of an elastic disc between two rigid plates. The frictional effects are clearly shown on the motion behavior of the disc and on the energy dissipation.

\section{The bi-potential method}

\subsection{Unilateral contact and Coulomb's friction laws}

First of all, some basic definitions and notations are set up. Let $\Omega_{1}$ and $\Omega_{2}$ be two bodies in contact at a point $\mathrm{M}$ for some value of the time (Fig. 1). The instantaneous velocity of the particles of $\Omega_{1}$ and $\Omega_{2}$ passing at point $\mathrm{M}$ and its projection point $\mathrm{M}^{\prime}$ being, respectively, $\dot{\mathbf{u}}_{1}$ and $\dot{\mathbf{u}}_{2}$,

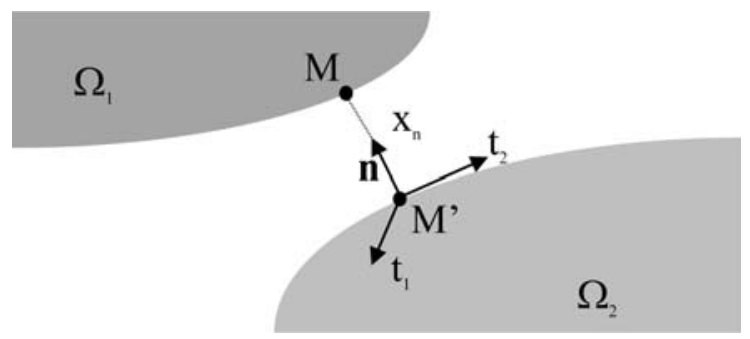

Fig. 1 Contact kinematics

where the superposed dot denotes the time derivative. The relative velocity is $\dot{\mathbf{u}}=\dot{\mathbf{u}}_{1}-\dot{\mathbf{u}}_{2}$. Let $\mathbf{r}$ be the contact reaction acting at $\mathrm{M}^{\prime}$ from $\Omega_{2}$ onto $\Omega_{1}$. Then $\Omega_{2}$ is subjected to the reaction $-\mathbf{r}$, acting from $\Omega_{1}$. Let $\mathbf{n}$ denote the normal unit vector at point $\mathbf{M}^{\prime}$ to the bodies, directed towards $\Omega_{1}$, and $\mathbf{T}\left(\mathrm{t}_{1}, \mathrm{t}_{2}\right)$ denotes the orthogonal plane to $\mathbf{n}$ in $\Re^{3}$. Any element $\dot{\mathbf{u}}$ and $\mathbf{r}$ may uniquely be decomposed in the form:

$\dot{\mathbf{u}}=\dot{\mathbf{u}}_{t}+\dot{\mathrm{u}}_{n} \mathbf{n}, \dot{\mathbf{u}}_{t} \in \mathbf{T}, \dot{\mathrm{u}}_{n} \in \Re$

$\mathbf{r}=\mathbf{r}_{t}+r_{n} \mathbf{n}, \quad \mathbf{r}_{t} \in \mathbf{T}, r_{n} \in \Re$

Classically, a unilateral contact law is characterized by a geometric condition of non-penetration, a static condition of no-adhesion and a mechanical complementarity condition. These three conditions are the so-called Signorini conditions written in terms of the signed contact distance $x_{\mathrm{n}}$ and the normal contact force $r_{n}$ :

$x_{n} \geq 0 ; \quad r_{n} \geq 0$ and $r_{n} x_{n}=0$

where $x_{\mathrm{n}}$ denotes the magnitude of the gap between the contact node and the target surface and is a violation of the contact compatibility:

$x_{n}=g+u_{n}$

with the initial gap:

$g=\left(\mathbf{x}_{1}-\mathbf{x}_{2}\right) \cdot \mathbf{n}$

where $\mathbf{x}_{1}$ and $\mathbf{x}_{2}$ denote respectively the coordinates of contact nodes of $\Omega_{1}$ and $\Omega_{2}$.

In the case of dynamic analysis such as impact problems, the Signorini's condition can be described in terms of velocity in conjunction with the sliding rule. At any time, the potential contact surfaces $\Gamma_{\alpha}(\alpha=1,2)$ can be split into two disjoint parts: $\Gamma_{\alpha}^{+}$where the bodies are already in contact $(g=0)$ and $\Gamma_{\alpha}^{-}$where the body are not in contact $(g>0)$. On $\Gamma_{\alpha}^{+}$, the unilateral contact conditions turns into

$u_{\mathrm{n}} \geq 0 ; r_{\mathrm{n}} \geq 0$ and $r_{\mathrm{n}} u_{\mathrm{n}}=0$ on $\Gamma_{\alpha}^{+}$

or in a rate form:

$\dot{\mathrm{u}}_{n} \geq 0 ; r_{n} \geq 0$ and $r_{n} \dot{\mathrm{u}}_{n}=0$ on $\Gamma_{\alpha}^{+}$

Let $K_{\mu}$ denote Coulomb's cone:

$K_{\mu}=\left\{\mathbf{r} \in \Re^{3}\right.$ such that $\left.\left\|\mathbf{r}_{\mathrm{t}}\right\| \leq \mu r_{\mathrm{n}}\right\}$

$K_{\mu}$ is a closed convex set. The complete contact law is a complex non-smooth dissipative law including three 
statuses: no contact, contact with sticking and contact with sliding. The resulting analytical transcripts yield two overlapped "if. . .then. . .else" statements:

$$
\begin{array}{lc}
\text { if } r_{n}=0, \text { then } \dot{\mathbf{u}}_{n}>0, & \text { ! no contact } \\
\text { else if } \mathbf{r} \in \mathbf{I}\left(K_{\mu}\right) \text {, then } \dot{\mathbf{u}}=0, & \text { ! sticking } \\
\text { else } r_{n}>0, \mathbf{r} \in B\left(K_{\mu}\right), \dot{\mathbf{u}}_{n}=0 \text { and } \exists \lambda \geq 0 \\
\quad \text { such that } \dot{\mathbf{u}}_{t}=-\lambda \frac{\mathbf{r}_{t}}{\left\|\mathbf{r}_{t}\right\|}
\end{array}
$$

where $I\left(K_{\mu}\right)$ and $B\left(K_{\mu}\right)$ represent respectively the interior and the boundary of $\left(K_{\mu}\right)$. The parameter $\lambda$ represents indeed the magnitude of the relative velocity.

An alternative statement is the inverse law:

$\begin{array}{ll}\text { if } \dot{u}_{n}>0, \text { then } \mathbf{r}=0, & \text { ! no contact } \\ \text { else if } \dot{\mathbf{u}}=0, \text { then } \mathbf{r} \in \mathbf{I}\left(K_{\mu}\right), & \text { ! sticking } \\ \text { else } \dot{\mathbf{u}} \in \mathbf{T}, r_{n}>0 \text { and } \mathbf{r}_{\mathrm{t}}=-\mu r_{n} \frac{\dot{\mathbf{u}}_{t}}{\left\|\dot{\mathbf{u}}_{t}\right\|} & \text { ! sliding }\end{array}$

De Saxcé and Feng [12] have proposed a more compact form of the contact law. They have shown that the contact law (8) is equivalent to

$$
\left[\dot{\mathbf{u}}_{t}+\left(\dot{\mathrm{u}}_{\mathrm{n}}+\mu\left\|\dot{\mathbf{u}}_{\mathrm{t}}\right\|\right) \mathbf{n}\right] \in \partial \bigcup_{K_{\mu}}(\mathbf{r})
$$

where $\bigcup_{K_{\mu}}(\mathbf{r})$ denotes the so-called indicator function of the closed convex set $K_{\mu}$ :

$\bigcup_{K_{\mu}}(\mathbf{r})= \begin{cases}0 & \text { if } \mathbf{r} \in \mathrm{K} \mu \\ +\infty & \text { otherwise }\end{cases}$

$\partial \bigcup_{K_{\mu}}(\mathbf{r})$ is the subdifferential of $\bigcup_{K_{\mu}}$ at $\mathbf{r}$.

The following contact bi-potential is obtained:

$b_{\mathrm{c}}(-\dot{\mathbf{u}}, \mathbf{r})=\bigcup_{\Re_{-}}\left(-\dot{\mathrm{u}}_{\mathrm{n}}\right)+\bigcup_{K_{\mu}}(\mathbf{r})+\mu r_{\mathrm{n}}\left|-\dot{\mathbf{u}}_{\mathrm{t}}\right|$

where $\Re_{-}$is the set of the negative and null real numbers. Then the contact laws (8) and (9) can be, respectively, written in compact forms of implicit subnormality rules or differential inclusion rules

$-\dot{\mathbf{u}} \in \partial_{\mathrm{r}} b_{\mathrm{c}}(-\dot{\mathbf{u}}, \mathbf{r}), \quad \mathbf{r} \in \partial_{-\dot{\mathbf{u}}} b_{c}(-\dot{\mathbf{u}}, \mathbf{r})$

\subsection{Local algorithm}

In order to avoid nondifferentiable potentials that occur in nonlinear mechanics, such as in contact problems, it is convenient to use the Augmented Lagrangian Method [913]. Let $\rho>0$ be chosen in a suitable range to ensure numerical convergence. It can be determined directly according to the reduced contact flexibility matrix [24] (see Appendix). Consider the flexibility matrix for one contact node:

$\mathbf{W}=\left(\begin{array}{ll}w_{n n} & w_{n t} \\ w_{t n} & w_{t t}\end{array}\right)$ the parameter $\rho$ is then defined by

$\rho=1 / \min \left(w_{\mathrm{nn}}, w_{t t}\right) \quad$ or

$\rho=1 / \eta$ with $\eta$ the smallest eigenvalue of $\mathbf{W}$

So, $\rho$ is not a user-defined factor, as opposed to the penalty factor. For the contact bi-potential $b_{c}$, given by (12), provided that $\dot{\mathbf{u}}_{\mathrm{n}} \geq 0$ and $\mathbf{r} \in K_{\mu}$, we have

$\forall \mathbf{r}^{\prime} \in K_{\mu}, \quad \rho \mu\left(r_{\mathrm{n}}^{\prime}-r_{\mathrm{n}}\right)\left|\dot{\mathbf{u}}_{t}\right|+[\mathbf{r}-(\mathbf{r}-\rho \dot{\mathbf{u}})] \cdot\left(\mathbf{r}^{\prime}-\mathbf{r}\right) \geq 0$

Taking into account the decomposition (1), the following inequality has to be satisfied:

$\forall \mathbf{r}^{\prime} \in K_{\mu}, \quad(\mathbf{r}-\tau) \cdot\left(\mathbf{r}^{\prime}-\mathbf{r}\right) \geq 0$

where the modified augmented contact force $\tau$ is defined by:

$\tau=\mathbf{r}-\rho\left[\dot{\mathbf{u}}_{t}+\left(\dot{u}_{\mathrm{n}}+\mu\left|\dot{\mathbf{u}}_{t}\right|\right) \mathbf{n}\right]$

The inequality (17) means that $\mathbf{r}$ is the projection of $\tau$ onto the closed convex Coulomb's cone:

$\mathbf{r}=\operatorname{proj}\left(\tau, K_{\mu}\right)$

For the numerical solution of the implicit equation (19), Uzawa's algorithm can be used, which leads to an iterative process involving one predictor-corrector step:

Predictor: $\quad \tau^{i+1}=\mathbf{r}^{\mathrm{i}}-\rho\left[\dot{\mathbf{u}}_{t}^{i}+\left(\dot{u}_{n}^{i}+\mu\left|\dot{\mathbf{u}}_{t}^{i}\right|\right) \mathbf{n}\right]$

Corrector: $\quad \mathbf{r}^{i+1}=\operatorname{proj}\left(\tau^{i+1}, K_{\mu}\right)$

It is worth noting that, in this algorithm, the unilateral contact and the friction are coupled via the contact bipotential. This approach may be compared with the augmented Lagrangian method developed by Jean and Touzot [25], and with the return mapping method developed by Giannakopoulos [26] and Wriggers et al. [27] in which the unilateral contact and the friction are not coupled. Another gist of the bi-potential method is that the corrector can be analytically found with respect to the three possible contact statuses: $\tau \in K_{\mu}$ (contact with sticking), $\quad \tau \in K_{\mu}^{*} \quad$ (no contact) and $\tau \in \Re^{3}-\left(K_{\mu} \cup K_{\mu}^{*}\right)$ (contact with sliding). $K_{\mu}^{*}$ is the polar cone of $K_{\mu}$ (Fig. 2).

This corrector step is explicitly given as follows:

$$
\begin{array}{lll}
\text { if } \quad \mu\left|\tau_{t}^{i+1}\right|<-\tau_{\mathrm{n}}^{i+1} \quad \text { then } \mathbf{r}^{i+1}=0 & \text { ! no contact } \\
\text { else if }\left|\tau_{t}^{i+1}\right| \leq \mu \tau_{n}^{i+1} \quad \text { then } r^{i+1}=\tau^{i+1} & \text { ! sticking } \\
\text { else } \quad \mathbf{r}^{i+1}=\tau^{i+1}-\frac{\left(\left|\tau_{t}^{i+1}\right|-\mu \tau_{\mathrm{n}}^{i+1}\right)}{1+\mu^{2}}\left(\frac{\tau_{i+1}^{i+1}}{\left|\tau_{t}^{i+1}\right|}+\mu \mathbf{n}\right) & \text { ! sliding }
\end{array}
$$

After the convergence of the predictor-corrector process, the reaction forces in the global frame are obtained by

$\mathbf{R}_{\mathrm{c}}=\mathbf{H}(\mathbf{u}) \mathbf{r}$

where $\mathbf{H}(\mathbf{u})$ is the mapping from the local frame to the global frame. 


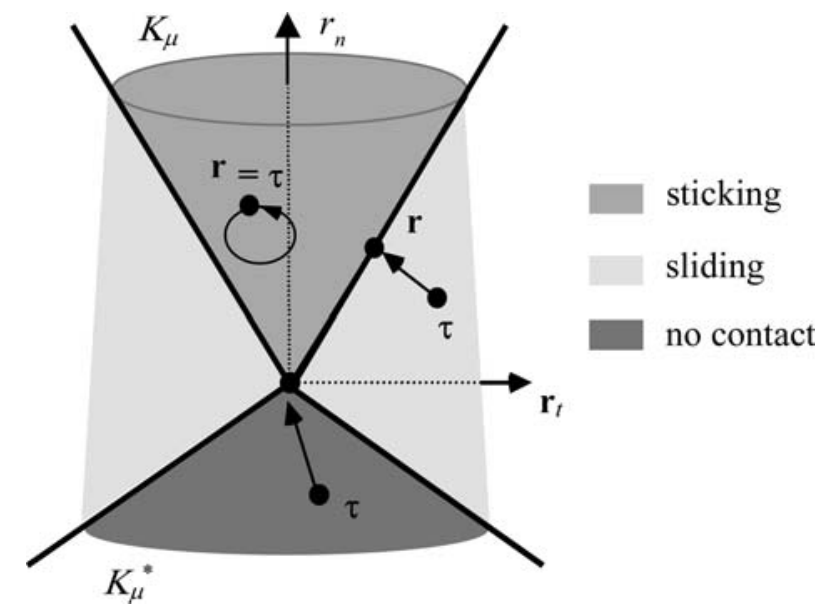

Fig. 2 Coulomb's cone $K_{\mu}$ and its polar one $K_{\mu}^{*}$

\section{Global integration algorithms}

Generally, non-linear dynamic mechanical behaviors of solid media with contact are governed by the dynamic equilibrium equation (after finite element discretisation):

$\mathbf{M} \ddot{\mathbf{u}}=\mathbf{F}+\mathbf{R}_{\mathrm{c}}$

where

$\mathbf{F}=\mathbf{F}_{\mathrm{ext}}+\mathbf{F}_{\text {int }}-\mathbf{C} \dot{\mathbf{u}}$

The vectors $\mathbf{F}_{\text {int }}$ and $\mathbf{F}_{\text {int }}$ denote, respectively, the internal and external forces. $\mathbf{R}_{\mathrm{c}}$ is the assembled vector of contact forces obtained from Eq. (23). $\mathbf{M}$ is the mass matrix and $\mathbf{C}$ the damping matrix. $\dot{\mathbf{u}}$ is the velocity vector and $\ddot{\mathbf{u}}$ the acceleration vector. It is noted that the stiffness effect is taken into account by the internal forces vector $\mathbf{F}_{\text {int }}$. The most common method for integrating the dynamics Eq. (24) is the Newmark method. It is based on the following assumptions concerning the relation between displacement, velocity and acceleration:

$\mathbf{u}^{t+\Delta t}=\mathbf{u}^{t}+\Delta t \dot{\mathbf{u}}^{t}+\Delta t^{2}\left[(0.5-\alpha) \ddot{\mathbf{u}}^{t}+\alpha \ddot{\mathbf{u}}^{t+\Delta t}\right]$

$\dot{\mathbf{u}}^{t+\Delta t}=\dot{\mathbf{u}}^{t}+\Delta t\left[(1-\beta) \ddot{\mathbf{u}}^{t}+\beta \ddot{\mathbf{u}}^{t+\Delta t}\right]$

The parameters $\alpha$ and $\beta$ determine the stability and precision of the algorithm. In standard applications without contact, the values corresponding to trapezoidal rule (with parameters $\beta=0.5$ and $\alpha=0.25$ ) are commonly used. A second order scheme ensures good stability and regularity of the solution. Chaudhary and Bathe [28] presented a solution method for dynamic analysis of $3 \mathrm{D}$ contact problems with friction. The Lagrange multiplier method and the Coulomb friction law were used to solve the constrained boundary conditions. The implicit time integration of the dynamic response was performed using the Newmark scheme with parameters $\beta=0.5$ and $\alpha=0.5$. A very small time step was used to ensure the energy and momentum balance criteria. However, in high-velocity impact problems, higher order approximation does not neces- sarily mean better accuracy, and may even be superfluous. At the moment of sudden change of contact conditions (impact, release of contact), the velocity and acceleration are not continuous, and excessive regularity constraints may lead to serious errors such as an unstable increase of the energy during the numerical simulations [15]. Moreau [19], Jean and Wronski [20, 21] have proposed a first order algorithm that is used in this work. Equation (24) can be transformed into

$\mathbf{M} \mathrm{d} \dot{\mathbf{u}}=\mathbf{F} \mathrm{d} t+\mathbf{R}_{\mathrm{c}} \mathrm{d} t$

We can now integrate Eq. (28) between consecutive time configuration $\mathrm{t}$ and $t+\Delta t$, using the following approximations:

$\int_{t}^{t+\Delta t} \mathbf{M} \mathrm{d} \dot{\mathbf{u}}=\mathbf{M}\left(\dot{\mathbf{u}}^{t+\Delta t}-\dot{\mathbf{u}}^{t}\right)$

$\int_{t}^{t+\Delta t} \mathbf{F} \mathrm{d} t=\Delta t\left[(1-\xi) \mathbf{F}^{t}+\xi \mathbf{F}^{t+\Delta t}\right]$

$\int_{t}^{t+\Delta t} \mathbf{R}_{\mathrm{c}} \mathrm{d} t=\Delta t \mathbf{R}_{\mathrm{c}}^{t+\Delta t}$

$\mathbf{u}^{t+\Delta t}-\mathbf{u}^{t}=\Delta t\left[(1-\theta) \dot{\mathbf{u}}^{t}+\theta \dot{\mathbf{u}}^{t+\Delta t}\right]$

where $0 \leq \xi \leq 1 ; 0 \leq \theta \leq 1$. In the iterative solution procedure, all the values at time $t+\Delta t$ are replaced by the values of the current iteration $i+1$; for example, $\mathbf{F}^{t+\Delta t}=\mathbf{F}^{i+1}$. A standard approximation of $\mathbf{F}^{i+1}$ gives

$$
\begin{aligned}
\mathbf{F}^{i+1} & =\mathbf{F}_{\text {int }}^{i}+\frac{\partial \mathbf{F}}{\partial \mathbf{u}}\left(\mathbf{u}^{i+1}-\mathbf{u}^{i}\right)+\frac{\partial \mathbf{F}}{\partial \dot{\mathbf{u}}}\left(\dot{\mathbf{u}}^{i+1}-\dot{\mathbf{u}}^{i}\right) \\
& =\mathbf{F}_{\text {int }}^{i}-\mathbf{K}^{i} \Delta \mathbf{u}-\mathbf{C}^{i} \Delta \dot{\mathbf{u}}
\end{aligned}
$$

where $\mathbf{K}$ and $\mathbf{C}$ are respectively the tangent stiffness matrix and the damping matrix.

Finally, we obtain the recursive form of Eq. (28) in terms of displacements

$\overline{\mathbf{K}}^{i} \Delta \mathbf{u}=\overline{\mathbf{F}}^{i}+\mathbf{R}_{\mathrm{c}}^{i+1}$

$\mathbf{u}^{i+1}=\mathbf{u}^{i}+\Delta \mathbf{u}$

where the so-called effective terms are given by

$\overline{\mathbf{K}}^{i}=\xi \mathbf{K}^{i}+\frac{\xi}{\theta \Delta t} \mathbf{C}^{i}+\frac{1}{\theta \Delta t^{2}} \mathbf{M}^{i}$

$\overline{\mathbf{F}}^{i}=(1-\xi)\left(\mathbf{F}_{\mathrm{int}}^{t}+\mathbf{F}_{\mathrm{ext}}^{t}\right)+\xi\left(\mathbf{F}_{\mathrm{int}}^{i}+\mathbf{F}_{\mathrm{ext}}^{t+\Delta t}\right)$

$$
-\frac{1}{\theta \Delta t^{2}} \mathbf{M}^{i}\left(\mathbf{u}^{i}-\mathbf{u}^{t}-\Delta t \dot{\mathbf{u}}^{t}\right)
$$

In this algorithm, the acceleration does not need to be computed. At the end of each time step, the velocity is updated by

$\dot{\mathbf{u}}^{t+\Delta t}=\left(1-\frac{1}{\theta}\right) \dot{\mathbf{u}}^{t}+\frac{1}{\theta \Delta t}\left(\mathbf{u}^{t+\Delta t}-\mathbf{u}^{t}\right)$ 
Eq. (34) is strongly non-linear, because of finite strains and large displacements of solid, for instance in large deformation problems. Besides, the constitutive law of contact with friction is usually represented by inequalities and the contact potential is even nondifferentiable. Instead of solving this equation in consideration of all nonlinearities at the same time, Feng and Domaszewski [29] have proposed a solution strategy that consists in separating the nonlinearities in order to overcome the complexity of calculation and to improve the numerical stability. As $\Delta \mathbf{u}$ and $\mathbf{R}_{\mathrm{c}}$ are both unknown, Eq. (34) cannot be directly solved. First, Eq. (34) is solved without considering the contact forces so as to determine the contact interpenetration. Then, the vector $\mathbf{R}_{\mathrm{c}}$ is determined by the bi-potential method (Eqs. 20-23) in a reduced system, which only concerns contact nodes. Then, the vector $\Delta \mathbf{u}$ can be computed in the whole structure, using contact reactions as external loading. This solution procedure involving contact modeling can be summarized in Box 1.

1. Read the data: mesh, material properties, boundary conditions, ...

2. Determine the mass matrix $\mathbf{M}$ and the damping matrix $\mathbf{C}$

3. For each time step

3.1. Determine the external force vector $\mathbf{F}_{\mathrm{ext}}$

3.2. Detect contact conditions (local frame, gap vector...)

3.3. For each Newton-Raphson equilibrium iteration

3.3.1. Compute the tangent stiffness matrix $\mathbf{K}$ and the internal force vector $\mathbf{F}_{\text {int }}$

3.3.2. Compute the effective stiffness matrix $\overline{\mathbf{K}}$ and the effective force vector $\overline{\mathbf{F}}$

3.3.3. Modify $\overline{\mathbf{K}}$ and $\overline{\mathbf{F}}$ for essential boundary conditions

3.3.4. Solve $\overline{\mathbf{K}} \Delta \mathbf{u}=\overline{\mathbf{F}}$

3.3.5. Compute reaction forces $\mathbf{R}_{\mathrm{c}}$ by local algorithm

3.3.6. Solve $\overline{\mathbf{K}} \Delta \mathbf{u}=\overline{\mathbf{F}}+\mathbf{R}_{\mathrm{c}}$

3.3.7. Actualize $\mathbf{u}=\mathbf{u}+\Delta \mathbf{u}$

3.3.8. Check convergence criteria, if not met, go to 3.3.1.

3.4. Compute the velocity vector

3.5. Gather element nodal displacement

3.6. Compute stresses and strains for each element and output

Box 1. Solution procedure

It is very important to note that, as opposed to the penalty method or Lagrange multiplier method, the bipotential method neither changes the global stiffness matrix, nor increases the degrees of freedom. One consequence of this interesting property is that it is easy to implement contact and friction problems in an existing general-purpose finite element code by this method. In

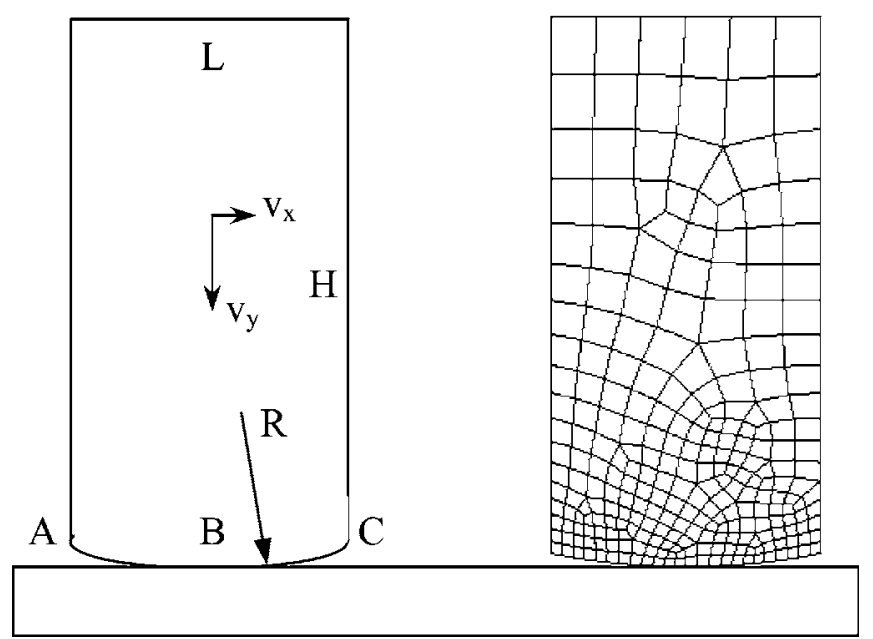

Fig. 3 Oblique impact of an elastic plate and finite element mesh

addition, the solution procedure is more stable because of the separation of nonlinearities and improved numerical algorithms for calculation of contact reactions.

\section{Numerical results}

The algorithms presented above have been implemented and tested in the finite element code FER/Impact. Many application examples, in static or quasi-static cases, have been carried out using the present method [24, 30, 31].

The first example of dynamics analysis will be presented to show the validity of the model developed. The problem concerns the oblique impact of a $2 \mathrm{D}$ elastic plate onto a rigid surface with rebounding. This example has been proposed and studied by Kim and Kwak [32] using Linear Complementarity Problem (LCP) formulation. The geometric configuration and finite element mesh are displayed in Fig. 3. Finer finite element meshes are generated near the contact surface so as to improve the accuracy on the contact modeling. A non-uniform mesh is thus used instead of a uniform one. A meshadaptive technique in contact mechanics has been developed by Wriggers and Scherf [33].

The characteristics of this example are: Young's modulus: $\mathrm{E}=10^{7} \mathrm{~Pa}$; Poisson's ratio: $v=0.25$; Mass density: $\rho=1000 \mathrm{~kg} / \mathrm{m}^{3}$; Friction coefficient: $\mu=0.2$; Initial velocity: $v_{x}=3 \mathrm{~m} / \mathrm{s}, v_{y}=-5 \mathrm{~m} / \mathrm{s}$; Geometric size: $L=0.04 \mathrm{~m}, H=0.08 \mathrm{~m}$, radius $R=0.101 \mathrm{~m}$, thickness $e=0.01 \mathrm{~m}$; Integration time: $3 \times 10^{-3} \mathrm{~s}$. The plate is modeled by 296 nodes and 259 linear quadrilateral plane stress elements (Fig. 3). The solution parameters are reported in Table 1.

Table 1 Solution parameters

\begin{tabular}{llll}
\hline Code & time step $\Delta t(\mathrm{~s})$ & $\begin{array}{l}\text { Integration } \\
\text { parameters }\end{array}$ & $\begin{array}{l}\text { Penalty } \\
\text { factor }\end{array}$ \\
\hline ANSYS & $10^{-5}$ (initial step) & $\begin{array}{c}\text { (Newmark) } \\
\alpha=0.25 \\
\theta=\xi=0.5\end{array}$ & $10^{5}$ \\
FER/Impact & $10^{-5}$ & no \\
\hline
\end{tabular}


Fig. 4 Evolution of reaction forces at point $\mathrm{B}$ versus time

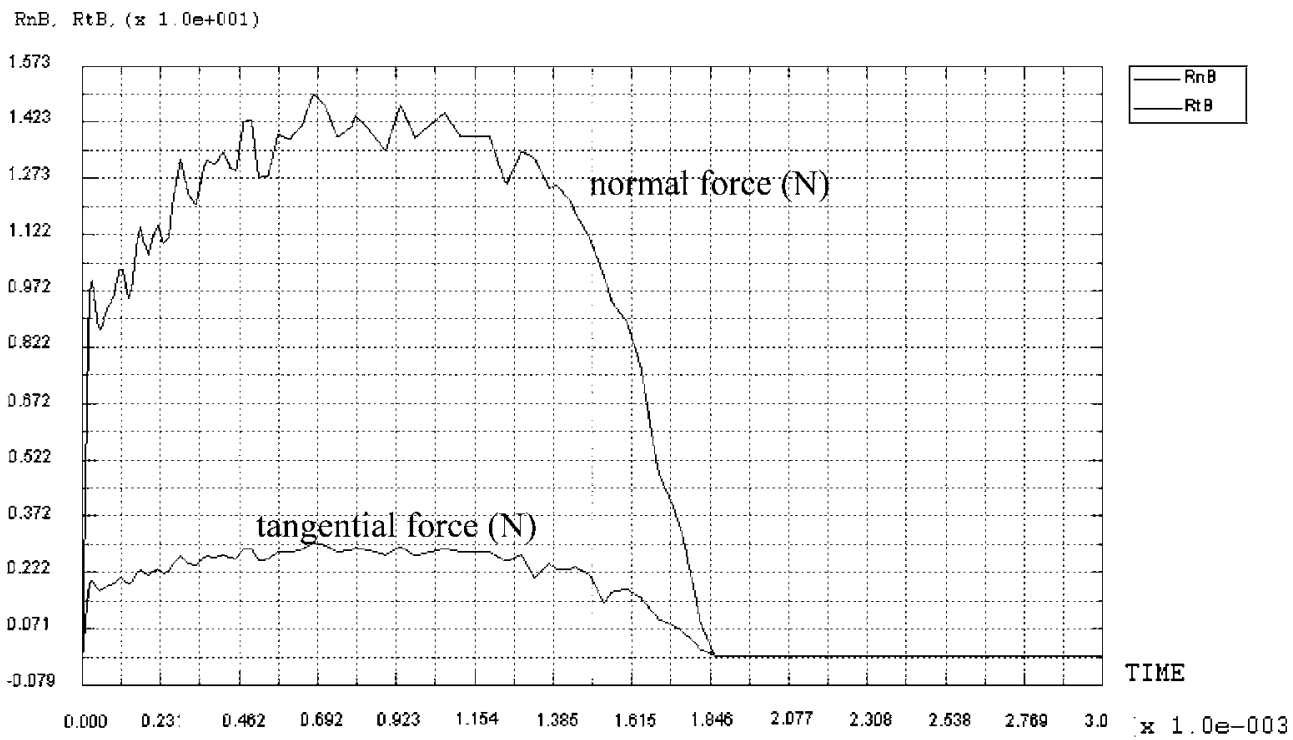

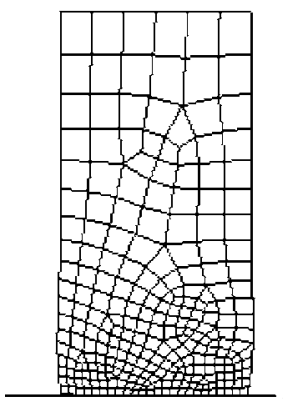

$\mathrm{t}=0.8 \mathrm{~ms}$

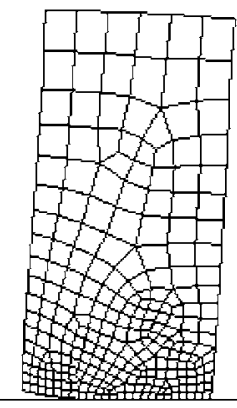

$\mathrm{t}=1.2 \mathrm{~ms}$

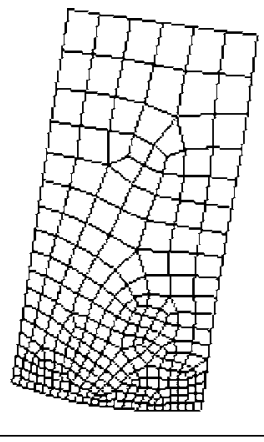

$\mathrm{t}=3 \mathrm{~ms}$
Fig. 5 Deformed meshes at different time steps

Figure 4 shows the evolution of contact and friction forces versus time at the center point $\mathrm{B}$ of arch $\mathrm{ABC}$. Figure 5 shows the deformed meshes at different times.
Table 2 Comparison between the codes

\begin{tabular}{llll}
\hline Code & Formulation & Contact algorithm & $t_{\mathrm{r}}(\mathrm{ms})$ \\
\hline ANSYS & Second order implicit & Penalty & 1.8 \\
SYSTUS & Second order implicit & Penalty & 1.9 \\
ABAQUS/ & Second order implicit & $\begin{array}{c}\text { Lagrange } \\
\text { multiplier }\end{array}$ & 1.8 \\
Standard & & Penalty & 1.8 \\
RADIOSS & Explicit & Penalty & 1.7 \\
LS-DYNA & Explicit & Bi-potential & 1.85 \\
FER/Impact & First order implicit & & \\
\hline
\end{tabular}

From Fig. 4, the rebounding occurred at $1.85 \times 10^{-3} \mathrm{~s}$. Figure 6 shows the evolution of Von Mises stress contours in the plate. The maximum value is $916500 \mathrm{~Pa}$. As shown in Figs. 5 and 6, the rebound is not in the normal direction of the rigid surface because of the friction effect and the oblique impact. The French IPSI workshop proposed this example as a test example [34]. Several industrial codes (ANSYS, ABAQUS, LS-DYNA,

Fig. 6 Evolution of Von Mises stress versus time

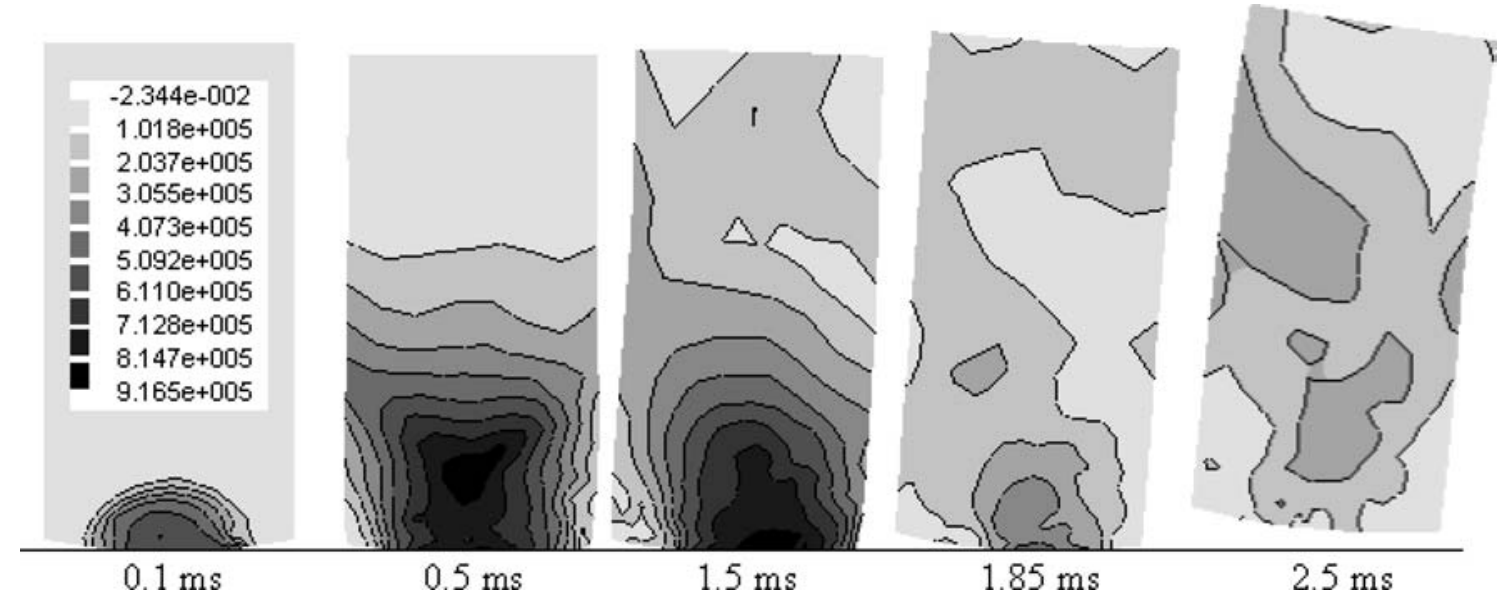




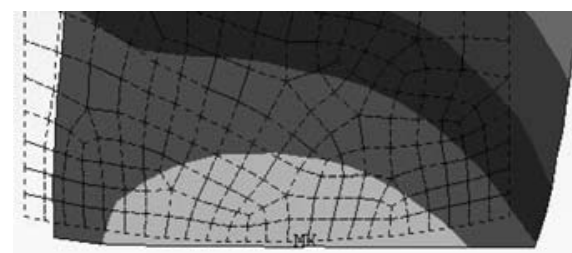

a) ANSYS

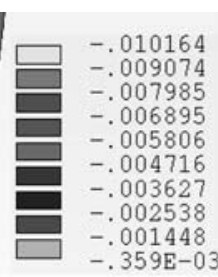

Fig. 7 Deformed meshes with isovalues of $U_{Y}$ at $t=1.0 \mathrm{~ms}$

Table 3 Performance of FER/Impact and ANSYS

\begin{tabular}{llll}
\hline Code & $\begin{array}{l}\text { Number } \\
\text { of time steps }\end{array}$ & $\begin{array}{l}\text { Iteration } \\
\text { number }\end{array}$ & CPU time (s) \\
\hline ANSYS & 766 & 7821 & 8733 \\
FER/Impact & 300 & 2423 & 1850 \\
\hline
\end{tabular}

SYSTUS, RADIOSS) have been used for analysis of this example. Table 2 summarizes the formulation used by each code and the instant $\left(t_{\mathrm{r}}\right)$ of rebounding of point $\mathrm{B}$. The comparison is rather homogeneous. In a more detailed investigation, we can observe significant differences between FER/Impact and ANSYS. Figure 7 shows the deformed shapes obtained by the two codes with zoom. We can see the penetration in the case of ANSYS, but not with FER/Impact. The performance of the present approach, as compared to ANSYS, is reported in Table 3 in which the iteration number is the cumulative Newton-Raphson iteration number necessary to achieve the analysis. These results show once again the efficiency of the proposed method. It is noted that the analysis is performed on a PC (Pentium III 733 $\mathrm{MHz}$ ).

Some test examples of contact problems in static case have been proposed by Feng [30]. We propose in this paper another test example of impact problems. This example simulates the impact of an elastic disc between two rigid plates. Dimensionless data are intentionally used. The disc has a diameter of 2 and a thickness of 1 . The Saint-Venant-Kirchhoff material model is assumed for the disc with material constants: $E=1000, v=0.45$, $\rho=1$. The disc is given an initial velocity of $v_{x}=2$ and $v_{y}=-2$, hitting the bottom plate and afterwards the top plate and so on. The total simulation time is 8 and the

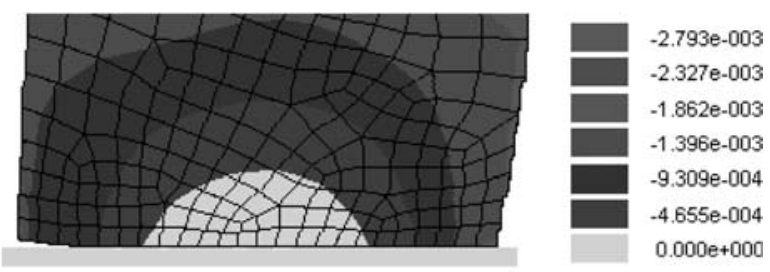

b) FER/Impact

solution parameters are taken as: $\Delta t=10^{-3}$, $\xi=\theta=0.5$. In order to show the frictional effects, both frictionless contact and frictional contact (with $\mu=0.5$ ) are considered. Fig. 8 shows the deformed meshes of the disc for both cases. It is quite easy to understand the situation of the frictionless case where the disc does not rotate and moves regularly from the left to the right as shown in Fig. 8 (a). However, in the case of frictional contact, the motion behavior is not predictable. After the first shock, the disc rotates due to the tangential friction forces. After the second shock, the rotation is inverted and the tangential friction forces make the disc move to the left. So the disc returns almost to its initial position as shown in Fig. 8 (b). Fig. 9 shows the distribution of the Von Mises stress of the disc when it hits the bottom plate at $t=1.588$. Without friction, the distribution is symmetric as expected, but this is not the case with friction. Once again, the frictional effects are apparently demonstrated. It is also interesting to examine the energy evolution. Figures 10 and 11 show the plots of the kinetic energy $E_{k}$, the elastic strain energy $E_{e}$ and the total energy $E_{t}$. We can observe clearly that the total energy is perfectly conserved in the case of frictionless contact (Fig. 10). However, in the case of frictional contact, the total energy decreases at each shock (Fig. 11). So the energy is dissipated by frictional effects as expected.

\section{Conclusion}

In this paper, we have presented the recent development of the bi-potential method applied to dynamic analysis in contact mechanics. The numerical algorithms have been described. The proposed algorithm is based on the local analysis of frictional contact problems by the bi-potential method and on the global resolution of the dynamic

Fig. 8 Deformed meshes $(t=0,1.588,3.888,6.258,7.498)$
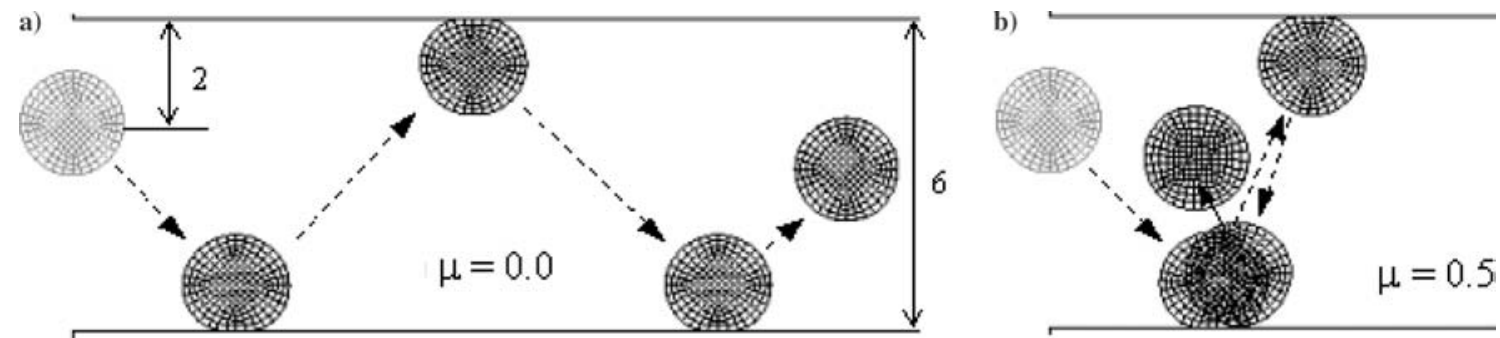
Fig. 9 Isovalues of Von Mises stress at $t=1.588$ a)

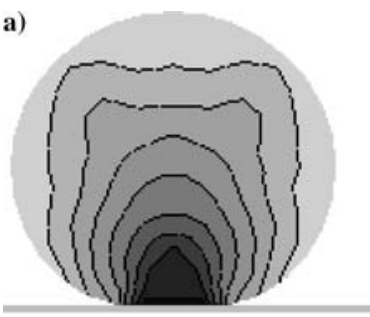

$\mu=0.0$ b)

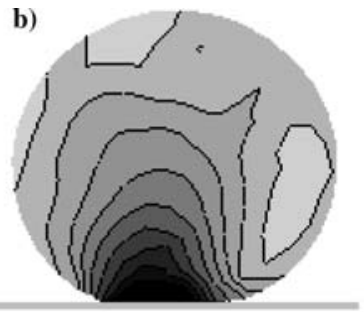

$\mu=0.5$
Mises

$0.000 \mathrm{e}+000$

$2.000 \mathrm{e}+001$

$4.000 \mathrm{e}+001$

$6.000 \mathrm{e}+001$

$8.000 \mathrm{e}+001$

$1.000 \mathrm{e}+002$

$200 \mathrm{e}+002$

$400 \mathrm{e}+002$

$.600+002$

$600 \mathrm{e}+002$

$.800 \mathrm{e}+002$

$2.000 \mathrm{e}+002$
Fig. 10 Energy evolution without friction $(\mu=0.0)$
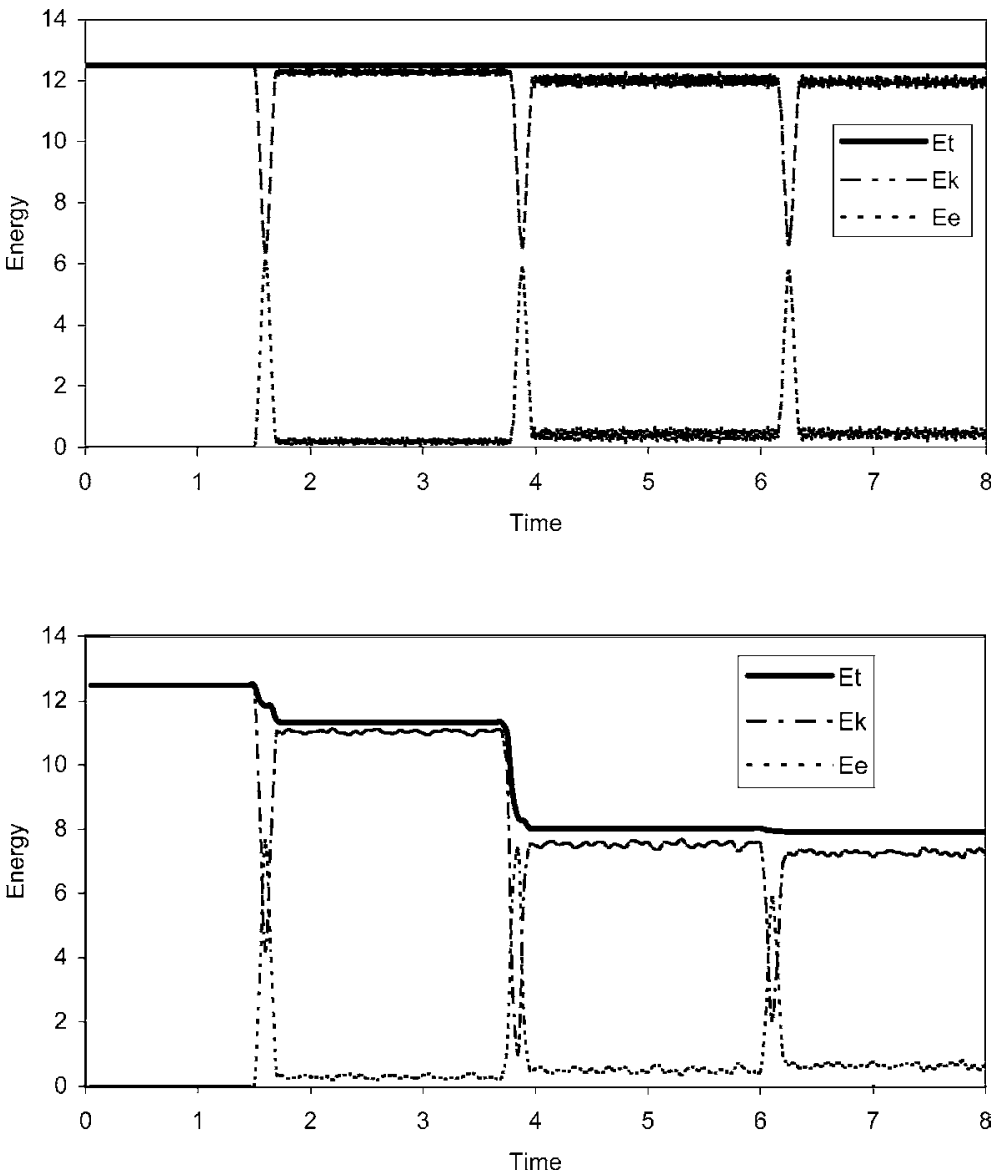

Fig. 11 Energy evolution with friction $(\mu=0.5)$ equation using the first order algorithm. Numerical results demonstrated that our model could provide better performance in terms of numerical stability and precision when compared to other existing formulations such as penalty and second order implicit integration schemes. The proposed algorithm not only preserves the energy conservation property of frictionless contact of solids exactly, but also determines the physical energy dissipation by frictional effects quantitatively.

\section{Appendix: computation of the flexibility matrix}

In order to reduce the dimension of the problem before the iterative procedure, one can use the condensation technique. Reformulating the structure equilibrium Eq. (34) under the following form:

$$
\left[\begin{array}{ll}
\overline{\mathbf{K}}_{r r} & \overline{\mathbf{K}}_{r c} \\
\overline{\mathbf{K}}_{r c}^{T} & \overline{\mathbf{K}}_{c c}
\end{array}\right]\left\{\begin{array}{c}
\Delta \mathbf{u}_{r} \\
\Delta \mathbf{u}_{c}
\end{array}\right\}=\left\{\begin{array}{c}
\overline{\mathbf{F}} \\
\mathbf{R}_{c}
\end{array}\right\}
$$

where $\Delta \mathbf{u}_{c}$ is the displacement vector of the contact node and $\Delta \mathbf{u}_{r}$ for the others. The condensed degrees $\Delta \mathbf{u}_{r}$ are eliminated by:

$\Delta \mathbf{u}_{r}=\overline{\mathbf{K}}_{r r}^{-1}\left(\overline{\mathbf{F}}-\overline{\mathbf{K}}_{r c} \Delta \mathbf{u}_{c}\right)$

Replacing (A.2) into (A.1), one obtains:

$\overline{\mathbf{K}}_{c c}^{*} \Delta \mathbf{u}_{c}=\overline{\mathbf{F}}^{*}+\mathbf{R}_{c}$

with 


$$
\begin{aligned}
\overline{\mathbf{K}}_{c c}^{*} & =\overline{\mathbf{K}}_{c c}-\overline{\mathbf{K}}_{r c}^{\mathrm{T}} \overline{\mathbf{K}}_{r r}^{-1} \overline{\mathbf{K}}_{r c} \quad \text { and } \\
\overline{\mathbf{F}}^{*} & =-\overline{\mathbf{K}}_{r c}^{\mathrm{T}} \overline{\mathbf{K}}_{r r}^{-1} \overline{\mathbf{F}}
\end{aligned}
$$

The contact flexibility matrix is thus obtained by

$\mathbf{W}_{c c}=\left(\overline{\mathbf{K}}_{c c}^{*}\right)^{-1}$

Acknowledgement. We gratefully thank the reviewers for their valuable comments on improvement of the manuscript.

\section{References}

1. Zhong ZH, Macherle J (1992) Static contact problems - a review. Engng. Comput. 9:3-37

2. Klarbring A (1993) Mathematical programming in contact problems. In: Aliabdali MH, Brebbia CA (eds) Computational methods in contact mechanics. Southampton: Computational Mechanics Publications, pp. 233-263

3. Wriggers P (1995) Finite element algorithms for contact problems. Arch Comput Meth Engng 2:1-49

4. Kikuchi N, Oden JT (1988) Contact problems in elasticity: A study of variational inequalities and finite elements. Philadelphia: SIAM

5. Zhong ZH (1993) Finite element procedures in contact-impact problems. Oxford University Press

6. Wriggers P (2002) Computational contact mechanics. John Wiley \& Sons

7. Kikuchi N, Oden JT (1984) Contact problems in elastostatics. In: Oden JT, Carey GF (eds) Finite Elements, 5. Englewood Cliffs, NJ: Prentice-Hall

8. Feng ZQ, Touzot G (1992) Analysis of two and three-dimensional contact problems with friction by a mixed finite element method. Revue Euro. Eléments Finis 1:441-459

9. Alart P, Curnier A (1991) A mixed formulation for frictional contact problems prone to Newton like solution methods. Comp Meth Appl Mech Engng 92:353-375

10. Simo JC, Laursen TA (1992) An augmented Lagrangian treatment of contact problems involving friction. Comput \& Struct 42:97-116

11. De Saxcé G, Feng ZQ (1991) New inequality and functional for contact with friction: The implicit standard material approach. Mech Struct \& Mach 19:301-325

12. De Saxcé G, Feng ZQ (1998) The bi-potential method: a constructive approach to design the complete contact law with friction and improved numerical algorithms. Math Comput Model. special issue "Recent Advances in Contact Mechanics » 28:225-245

13. Klarbring A (1992) Mathematical programming and augmented Lagrangian methods for frictional contact problems. In: Curnier A (ed) Contact Mechanics Int Symp PPUR

14. Christensen PW, Klarbring A, Pang JS, Strömberg N (1998) Formulation and comparison of algorithms for frictional contact problems. Int J Num Meth Engng 42:145-173

15. Armero F, Petocz E (1998) Formulation and analysis of conserving algorithms for frictionless dynamic contact/impact problems. Comp Meth App. Mech Engng. 158:269-300
16. Laursen TA, Chawla V (1997) Design of energy conserving algorithms for frictionless dynamic contact problems. Int $\mathbf{J}$ Num Meth Engng 40:863-886

17. Laursen TA, Love GR (2002) Improved implicit integrators for transient impact problems - geometric admissibility within the conserving framework. Int J Num Meth Engng 53:245-274

18. Zienkiewicz OC, Wood WL, Taylor RL (1980) An alternative single-step algorithm for dynamic problems. Earthg Engng Struct Dyn 8:31-40

19. Moreau JJ (1988) Unilateral contact and dry friction in finite freedom dynamics. In: Moreau JJ, Panagiotopoulos PD (ed), Nonsmooth Mechanics and Applications, CISM Courses and Lectures 302:1-82

20. Jean M (1989) Dynamics with partially elastic shocks and dry friction: double scale method and numerical approach. In: Proc. 4d Meeting on unilateral problems in structural analysis, Capri

21. Wronski M, Jean M (1995) Some computational aspects of structural dynamics problems with frictional contact. In: Raous M, Jean M, Moreau JJ (eds) Contact Mechanics. Plenum Press, 137-144

22. Parisch H (1989) A consistent tangent stiffness matrix for threedimensional non-linear contact analysis. Int $\mathbf{J}$ Num Meth Engng 28:1803-1812

23. Kohnke P (1999) ANSYS Theory reference. Release 5.6 ANSYS inc

24. Feng ZQ (1991) Contribution à la modélisation des problèmes non linéaires: Contact, Plasticité et Endommagement, Ph.D. thesis, Université de Technologie de Compiègne, France

25. Jean M, Touzot G (1988) Implementation of unilateral contact and dry friction in computer codes dealing with large deformation problems. J Theo Appl Mech 7:145-160

26. Giannakopoulos AE (1989) The return mapping method for the integration of friction constitutive relations. Comput Struct $32: 157-167$

27. Wriggers P, Vu Van T, Stein E (1990) Finite element formulation of large deformation impact-contact problems with friction. Comput Struct 37:319-331

28. Chaudhary AB, Bathe KJ (1986) A solution method for static and dynamic analysis of three-dimensional contact problems with friction. Comput Struct 24:855-873

29. Feng ZQ, Domaszewski M (1995) Coupled treatment of frictional contact problems in a reduced system and applications in large deformation context. In: Raous M, Jean M, Moreau JJ (eds) Contact Mechanics, Plenum Press, 169-172

30. Feng ZQ (1998) Some test examples of 2D and 3D contact problems involving Coulomb friction and large slip, Math Comput Model special issue "Recent Advances in Contact Mechanics » 28:469-477

31. Feng ZQ, Peyraut F, Labed N (2003) Solution of large deformation contact problems with friction between Blatz-Ko hyperelastic bodies. Int J Engng Sci 41:2213-2225

32. Kim J, Kwak B (1996) Dynamic analysis of two-dimensional frictional contact by linear complementarity problem formulation. Int J Solids and Structures 33:4605-4624

33. Wriggers P, Scherf O (1995) An adaptive finite element technique for nonlinear contact problems. In: Raous $\mathrm{M}$, Jean $\mathrm{M}$, Moreau JJ (ed), Contact Mechanics Plenum Press, 183-194

34. IPSI Forum $\Phi^{2}$ AS (1999) Traitement des contacts en implicite et explicite, autour des tests, Volume XXIII, $\mathrm{N}^{\circ} 1$ bis 